\title{
Urinary polycyclic aromatic hydrocarbon metabolite associations with biomarkers of inflammation, angiogenesis, and oxidative stress in pregnant women
}

\author{
Kelly K. Ferguson', Thomas F. McElrath², Gerry G. Pace ${ }^{3}$, David Weller ${ }^{3}$, Lixia Zeng ${ }^{4}$, \\ Subramaniam Pennathur ${ }^{4}$, David E. Cantonwine ${ }^{2}$, and John D. Meeker ${ }^{5,{ }^{*}}$ \\ ${ }^{1}$ Epidemiology Branch, National Institute of Environmental Health Sciences, Research Triangle \\ Park, North Carolina, 27709, USA \\ 2Division of Maternal Fetal Medicine, Department of Obstetrics and Gynecology, Brigham and \\ Women's Hospital, Harvard Medical School, Boston, Massachusetts, 02115, USA \\ ${ }^{3} \mathrm{NSF}$ International, Ann Arbor, Michigan, 48105, USA \\ ${ }^{4}$ Division of Nephrology, Department of Medicine, University of Michigan, Ann Arbor, Michigan, \\ 48109, USA
}

${ }^{5}$ Department of Environmental Health Sciences, University of Michigan School of Public Health, Ann Arbor, Michigan, 48109, USA

\section{Abstract}

Environmental exposure to polycyclic aromatic hydrocarbons (PAHs) is prevalent and may adversely impact pregnancy and development of the fetus. The purpose of this exploratory study was to examine urinary PAH metabolites in association with mediators of these outcomes. To do so we measured a panel of 12 inflammatory, angiogenic, and oxidative stress biomarkers in plasma or urine from women in their third trimester of pregnancy $(n=200)$. Urinary PAH metabolites were highly detectable $(>88 \%)$ in the study population, and most were higher in women who had lower education levels, higher body mass index, and who were African American. Some PAH metabolites showed consistent positive associations with the plasma inflammation marker Creactive protein (CRP) and the urinary oxidative stress markers 8-hydroxydeoxyguanosine (8$\mathrm{OHdG}$ ) and 8-isoprostane. For example, an interquartile range increase in 2-hydroxynapthalene was associated with a 35\% increase in CRP (95\% confidence interval $=-0.13,83.2)$, a $14 \%$ increase in $8-\mathrm{OHdG}(95 \%$ confidence interval $=0.59,30.1)$, and a $48 \%$ increase in 8 -isoprostane $(95 \%$ confidence interval $=16.7,87.0)$. These data suggest that exposure to PAHs may cause systemic changes during pregnancy that could lead to adverse pregnancy or developmental outcomes; however, these results should be corroborated in a larger study population.

\section{Graphical abstract}

Correspondence to: John D. Meeker, meekerj@umich.edu, 734-764-7184.

Supporting Information Available: Correlations between PAHs, distributions of PAHs by demographic factors, correlations between biomarkers of inflammation, oxidative stress, and angiogenesis, distributions of plasma tyrosine adducts, associations between inflammation markers and PAHs, associations between angiogenic markers and PAHs, and associations between oxidative stress markers and PAHs. 


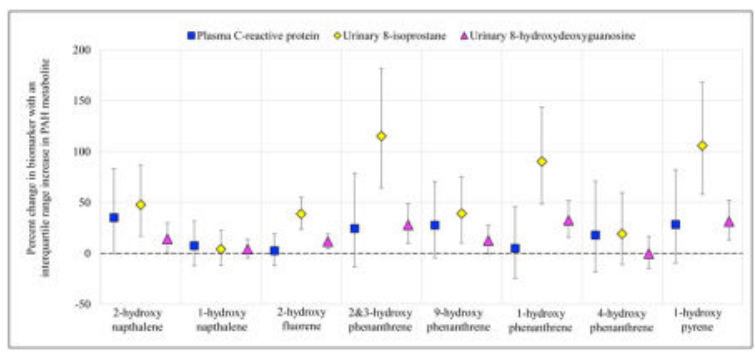

\section{INTRODUCTION}

Exposure to polycyclic aromatic hydrocarbons (PAHs) has become an important human health concern. These compounds are formed during the combustion of organic materials, are released into the air, and can eventually deposited into soil and water. ${ }^{1}$ Tobacco smoke is a major source of exposure, especially in smokers, but ambient air contamination with PAHs also occurs from industrial sites like coal-fired plants, automobiles, and waste facilities that burn garbage. ${ }^{1}$ Heavy air contamination can also occur naturally during forest fires or volcano eruptions. Residential wood fires for heating or cooking may be a source of exposure. Finally, dietary exposures may be a major source in the general population. Contamination of grains, vegetables, and dairy has been demonstrated in farm settings; fish accumulate PAHs; and grilling meats and vegetables results in high concentrations. ${ }^{2}$ Consequently, detection in the US and elsewhere is prevalent, as evidenced by personal and ambient air monitoring studies, DNA adducts in blood and tissue, and urinary metabolites. ${ }^{3-6}$

Some PAHs have demonstrated carcinogenicity, particularly benzo[a]pyrene. ${ }^{7,8}$ However exposure has been linked to other adverse disease endpoints as well, including inflammation of the eye, skin, and respiratory tract following acute exposure. ${ }^{8}$ Furthermore, prenatal exposure has been associated with fetal growth restriction, ${ }^{9}$ reduced gestational length, ${ }^{10}$ impaired neurodevelopment, ${ }^{11-13}$ and childhood asthma ${ }^{14,15}$ The majority of these studies have utilized personal or ambient air monitoring to assess exposure, which does not account for exposure through dietary sources. Within the context of pregnancy, very few studies have examined urinary biomarkers of PAH exposure, which reflect total exposure through multiple pathways.

In this exploratory study our objective was to examine the relationship between urinary hydroxylated PAH metabolite concentrations and biomarkers of mechanistic intermediates that may play an important role in the pathology of adverse pregnancy and developmental outcomes. To do so, we assessed associations between urinary PAH metabolites and a panel of 12 inflammatory, angiogenic, and oxidative stress biomarkers in a population of pregnant women from the Boston area. To our knowledge, this is the first study to date to examine the relationship between urinary PAHs and each of these biomarkers of mechanism during gestation. 


\section{MATERIALS AND METHODS}

\section{Study population}

Beginning in 2006, women were recruited early in pregnancy at Brigham and Women's Hospital in Boston, MA, as part of the ongoing LIFECODES longitudinal cohort study. Inclusion criteria were: 1) recruitment prior to 15 weeks gestation; 2) maternal age >18; and 3 ) intention to deliver at Brigham and Women's Hospital. As part of the study design, women provide urine samples for biomarker assessment at four visits during pregnancy (10, 18, 26 and 35 weeks gestation) which are stored at -80 degrees Celsius for future biomarker assessment. Additionally, demographic (age, race/ethnicity, education level, etc.) and questionnaire information (pregnancy history, alcohol and tobacco use, etc.) is obtained at the first study visit. Between 2006 and 2008, approximately 1600 women were recruited under funding by Abbott Diagnostics to investigate predictors of preeclampsia. ${ }^{16}$ Written informed consent was obtained from all study participants, and the study protocol was approved by the institutional review board of Brigham and Women's Hospital. For the present analysis, we selected all participants who had a spontaneous or placental preterm birth from within this population $(\mathrm{n}=76)$ as well as 124 random participants with term deliveries. Urine and plasma samples from the third study visit (median 26 weeks gestation) were utilized to assess biomarkers of exposure to PAH and of inflammation, angiogenesis, and oxidative stress, as our previous work demonstrated that this may be a sensitive window for exposure to environmental chemicals during pregnancy. ${ }^{17}$

\section{PAH biomarkers}

Urinary PAH metabolites were measured by isotope dilution-liquid chromatography with tandem mass spectrometry (LC-MS/MS) at NSF International (Ann Arbor, MI, USA). The metabolites measured included: 1-hydroxynapthalene (1-NAP); 2-hydroxynapthalene (2NAP); 2-hydroxyfluorene (2-FLU); 1-hydroxyphenanthrene (1-PHE); 2 and 3hydroxyphenanthrene, quantitated together (2\&3-PHE); 4-hydroxyphenanthrene (4-PHE); 9hydroxyphenanthrene (9-PHE); and 1-hydroxypyrene (1-PYR). The in-house LCMS method was developed and validated based on the approach previously described by Onyemauwa et al. (2009). ${ }^{18}$ Samples underwent enzymatic deconjugation of glucuronidated species, online solid phase extraction, and analysis with a Thermo Scientific (Waltham, MA, USA) Vantage triple quadrupole mass spectrometer using multiple reaction monitoring in negative ionization mode. Urine sample online extraction was performed using a Thermo Scientific Cyclone P 0.5 x $50 \mathrm{~mm}$ turbulent flow extraction column followed by chromatographic separation using a Waters (Milford, MA, USA) Xbridge C18 $5 \mu \mathrm{m}(3.0 \times 150 \mathrm{~mm})$ analytical column.

The precision of the method was determined by calculating the percent relative standard deviation (\%RSD) of repeated measurements of the quality control (QC) materials throughout the validation. This value reflects both the intra-day and inter-day variability of the assay. The \%RSD range for QC samples was $2.4-12 \%$. The method accuracy was obtained through 6 replicate analyses of analytes spiked at three different concentrations in human urine across validation runs on 3 separate days. The percent nominal concentration range across all analytes was $89-103$ percent. 
The limits of detection (LODs) were established by replicate injections of low concentration standards. The reported LOD is also the run limit of quantitation, and this level was always included as the low level standard in the calibration curve. The calibration curve response (analyte/internal standard area ratios) versus concentration were evaluated for goodness of fit. All validated analyte correlation coefficients $\left(\mathrm{R}^{2}\right)$ were $>0.994$. The LODs for hydroxylated PAHs were $50 \mathrm{ng} / \mathrm{L}$ for naphthalene metabolites and $10 \mathrm{ng} / \mathrm{L}$ for all other metabolites, which is similar to those reported by the Centers for Disease Control and Prevention for methods used in the National Health and Nutrition Examination Survey. ${ }^{5}$ Values that were reported as "below the LOD" were replaced with the LOD divided by the square root of $2 .{ }^{19}$ Values below the LOD that were reported numerically were kept as is. Urinary 1-NAP and 2-NAP concentrations were over the upper limit of detection for one study participant; levels of those metabolites were omitted from the present analysis.

For examining distributions of PAH metabolites we corrected concentrations for urine dilution with the following formula: $\mathrm{PAH}_{\mathrm{c}}=\mathrm{PAH}[(1.014-1) /(\mathrm{SG}-1)]$, where $\mathrm{PAH}_{\mathrm{c}}$ represents the corrected concentration, PAH represents the uncorrected urinary PAH concentration, SG represents the specific gravity of the specific sample, and 1.014 is the median urinary specific gravity of the samples. ${ }^{20}$ For all statistical models, uncorrected PAH metabolites were modeled with specific gravity included as a covariate.

\section{Inflammation, angiogenesis, and oxidative stress biomarkers}

We examined three classes of biomarkers, as listed in Table 1, in order to capture maternal inflammation, angiogenic factors, and oxidative stress in pregnancy. We chose these three groups because the strongest evidence of PAH effects from animal and cellular studies links exposure to oxidative stress and inflammation, and because human studies suggest a link between exposure and poor placentation which would be reflected by abnormalities in angiogenic biomarkers. We chose to examine multiple markers within each class since the various markers within a mechanistic group may represent different pathways.

Measurement of the plasma inflammation markers is described in detail elsewhere. ${ }^{21}$ Briefly, C-reactive protein (CRP) was measured in duplicate by enzyme-linked immunoassay, and a panel of inflammatory cytokines (IL-1 $\beta$, IL-6, IL-10, and TNF-a) were measured with a Milliplex MAP High Sensitivity Human Cytokine Magnetic Bead Panel (EMD Millipore Corporation) with a Luminex L200 instrument (Luminex, Austin, TX). These analyses were performed at the University of Michigan Cancer Center Immunological Monitoring Core (Ann Arbor, MI).

Soluble FMS-like tyrosine kinase 1 (sFlt-1) and placental growth factor (PIGF) are commonly measured biomarkers of angiogenesis that are somewhat useful in the prediction of preeclampsia, a disease characterized at least in part by poor implantation of the placenta. PIGF promotes angiogenesis, while sFlt-1 inhibits it. Total PIGF and free sFlt-1 were measured by Abbott Laboratories (Abbot Park, IL) using the ARCHITECT immunoassays. ${ }^{16}$

We measured oxidative stress biomarkers to assess oxidative DNA, lipid, and protein damage. 8-hydroxydeoxyguanosine (8-OHdG) and total 8-isoprostane, indicators of DNA 
and lipid damage, respectively, were measured in urine samples using enzyme immunoassay by Cayman Chemical Company (Ann Arbor, MI). ${ }^{22}$ To assess oxidative protein damage, we measured 3-nitrotyrosine (NY), 3-chlorotyrosine (ClY), and $o, o^{\prime}$-dityrosine (diY) in plasma samples, using methods described elsewhere. ${ }^{23}$ Briefly, plasma protein was precipitated from plasma samples and diluted using a phosphate buffer. Plasma was delipidated and then spiked with isotopically labeled internal standards, and the samples were hydrolyzed for 24 hours. Oxidized amino acids were quantified using liquid chromatography (LC) electrospray ionization tandem mass spectrometry (MS/MS) with multiple reaction monitoring MS/MS positive ion acquisition mode using an Agilent 6410 triple quadrupole MS system equipped with an Agilent 1200 LC system (Agilent Technologies, Santa Clara, CA). The coefficient of variation using pooled samples was noted to be $<5 \%$ for all oxidized amino acids measured.

\section{Statistical methods}

First, we tabulated demographic characteristics for factors that were considered as covariates for subsequent analyses, including: race/ethnicity (White, African-American, Other); education level (high school or less, technical school, junior college or some college, college graduate or above); health insurance provider (private, public); body mass index (BMI) at visit 1 of pregnancy $\left(25 \mathrm{~kg} / \mathrm{m}^{2}, 25-30 \mathrm{~kg} / \mathrm{m}^{2},>30 \mathrm{~kg} / \mathrm{m}^{2}\right)$; tobacco use in pregnancy (no smoking, some smoking); alcohol use in pregnancy (no alcohol use, some alcohol use); parity (nulliparous, parous); fetal sex (male, female); preterm birth (no, yes). Second, we examined distributions of specific gravity corrected urinary PAH metabolites and protein damage markers which have not been previously published, using geometric means and geometric standard deviations as well as selected percentiles. Third, we examined Spearman correlations between urinary PAH metabolites corrected for urinary specific gravity and between outcome biomarkers. Fourth, we examined associations between the biomarkers measured and demographic characteristics by creating linear regression models in which one marker was modeled in association with one demographic factor. Finally, we built linear regression models with one outcome biomarker regressed on one PAH metabolite, with adjustment for urinary specific gravity and demographic factors that were significantly associated with both exposure and outcome and influenced effect estimates by greater than 10 percent. For all statistical models biomarkers were natural log transformed so that their distributions approximated normality. All statistical analyses were performed using $\mathrm{R}$ version 3.2.3.

\section{RESULTS}

Demographic characteristics of the study population are similar to those observed in the greater LIFECODES birth cohort study (Table 2) ${ }^{21}$ Mothers were predominantly White, well-educated, had private as opposed to public health insurance providers (i.e., high socioeconomic status), and only few used tobacco or alcohol in pregnancy.

\section{PAH metabolite concentrations in pregnant women}

Distributions of specific gravity-corrected urinary PAH metabolite concentrations are presented in Table 3. For one study participant, the 2-NAP concentration was over the upper limit of detection, and was treated as missing for the presentation of results. Similarly for 
this subject there was an outlying concentration of 1-NAP, and this was likewise treated as missing. Overall, urinary PAH metabolites were highly detectable (>88\% detected for all metabolites) and distributions similar to those observed in the US National Health and Nutrition Examination Survey. ${ }^{24}$ Spearman correlations of specific gravity corrected urinary phthalate metabolites (Table S1) were moderate.

Urinary PAH metabolites were demonstrably different by some demographic factors (Table 4; concentrations by subgroup presented in Table S2). Most PAH were significantly higher in participants who had public as opposed to private health insurance, and in subjects who had lower education levels (high school or less). Concentrations of 2-FLU and 1-PYR were significantly higher in subjects who smoked or used alcohol during pregnancy, and who had $>30 \mathrm{~kg} / \mathrm{m}^{2} \mathrm{BMI}$ at the first study visit. Few differences were observed by race/ethnicity, although African-American and other race/ethnic groups had significantly higher urinary concentrations of 2-NAP and 1-PYR. No associations were observed between exposure and parity, fetal sex or preterm birth.

\section{Biomarkers of inflammation, angiogenesis, and oxidative stress in pregnant women}

We previously examined associations between inflammatory cytokines and demographic characteristics in a study population that included the participants from the present analysis, and thus do not present associations here. ${ }^{21}$ Spearman correlations between biomarkers within the present study population were low to moderate (Table S3). Within the present population, plasma CRP concentrations were significantly higher in African-American compared to White participants, and in subjects with high $\left(>30 \mathrm{~kg} / \mathrm{m}^{2}\right)$ compared to low $\left(<25 \mathrm{~kg} / \mathrm{m}^{2}\right)$ BMI at the first study visit (Table 5). IL-6 concentrations were significantly higher in Other race/ethnic groups compared to Whites, in participants with public compared to private health insurance providers, and in preterm births compared to term. Angiogenesis biomarker distributions have also been presented previously. ${ }^{25}$ In the present population no associations were observed with demographic factors (Table 5), although in the parent casecontrol study PIGF levels were significantly higher in African-American and Other race/ ethnic groups compared to Whites, in participants with public compared to private health insurance, in subjects with low compared to high BMI, and in parous and term pregnancies compared to nulliparous and preterm, respectively. ${ }^{25}$ sFlt- 1 concentrations were also higher in low compared to high BMI participants, and in nulliparous compared to parous mothers. ${ }^{25}$

Associations between the 8-OHdG and 8-isoprostane biomarkers and populations characteristics have been previously published as well, ${ }^{22}$ and are shown for the present study population in Table 5 also. In this exploratory analysis we additionally analyzed three biomarkers of oxidative protein damage that have not been previously examined in this study population. No associations were observed between any of the 3 markers and demographic factors in this study, except for slightly elevated ClY concentrations in parous compared to nulliparous mothers (Table 5; concentrations by subgroup presented in Table S4).

\section{PAH metabolite associations with biomarkers}

Results from models of urinary PAH metabolites in relation to biomarkers of inflammation, angiogenesis, and oxidative stress are presented in Figures 1-3, respectively (effect estimates 
and 95\% confidence intervals, CI, presented in Tables S5, S6, and S7). Models were adjusted for covariates that were associated with both exposure and outcome and changed effect estimates by $>10 \%$ for multiple PAH. Models were built using CRP for inflammation, sFlt-1 for angiogenesis markers, and 8-iso for oxidative stress, based on the most common associations with these biomarkers in crude models. Effect estimates were similar in crude and adjusted models, with slight attenuation in the latter; hence adjusted results only are presented here.

Inflammation models were adjusted for maternal race/ethnicity, education level, health insurance provider, and BMI at visit 1 . The largest effect estimates were observed in association with CRP concentrations. An interquartile range (IQR) change in 2-NAP, 2\&3PHE, 9-PHE, and 1-PYR concentrations were associated with 25-35\% percent increases in CRP. Some inverse associations were observed with the inflammatory cytokines measured. Particularly, 2\&3-PHE and 4-PHE concentrations were associated with lower levels of IL-1 $\beta$, IL-10, and TNF- $a$. Associations with IL-6 levels were very close to the null for all urinary PAH metabolites.

Models of angiogenesis biomarkers were adjusted for the same covariates as in the inflammation models. Lower levels of PlGF are thought to be adverse, as this is a proangiogenic protein, and higher levels of sFlt-1 are thought to be adverse, as the molecules bind pro-angiogenic factors and inhibit their activity. Increased 2-NAP, 2\&3-PHE, and 4PHE were associated with lower levels of PIGF. 9-PHE was associated with increases in sFlt-1.

Finally, oxidative stress models were adjusted for maternal age, education level, health insurance provider, and BMI at the first study visit. For naphthalene metabolites, 2-NAP was associated with increases in 8-OHdG and 8-iso, but not with protein oxidation biomarkers; however, 1-NAP was associated with increases in $\mathrm{ClY}$ and $\mathrm{DiY}$ protein oxidation biomarkers but not with 8-OHdG or 8-iso. 2-FLU was associated with increased 8-OHdG and 8-iso, but not with protein markers. For the phenanthrenes, a similar pattern was observed where a metabolite was associated with either $8-\mathrm{OHdG}$ and 8 -iso or protein oxidation markers, but not both. 2\&3-PHE, 9-PHE, and 1-PHE were associated with increased 8-OHdG and 8-iso, and the effect estimates were quite large for 8-iso (IQR change in 2\&3-PHE or 1-PHE associated with $90 \%$ or $115 \%$ increase in 8 -iso, respectively). 4-PHE was associated with increased ClY and DiY. Finally, 1-PYR was associated with increased 8-OHdG and 8-iso (IQR change associated with $106 \%$ increase in 8 -iso), but not with protein oxidation markers.

\section{DISCUSSION}

In an exploratory study of 200 pregnant women from the Boston area, we detected a number of associations between urinary PAH metabolites and biomarkers of inflammation, angiogenesis, and oxidative stress that may predict adverse downstream pregnancy outcomes. Associations differed by PAH metabolite and by outcome biomarker within each class; however interesting patterns emerged. 
Focusing on inflammation markers, we observed associations that were greatest in magnitude between CRP and 2-NAP, 9-PHE, and 1-PYR. For other inflammation biomarkers we observed unexpected inverse associations (particularly for 4-PHE and IL-1 $\beta$, IL-10, and TNF-a). This could reflect an immunosuppressive effect, which has been evidenced for some PAHs in animal studies. ${ }^{26,27}$

No individual PAHs were clearly associated with an adverse angiogenic marker profile of increased sFlt-1 and decreased PIGF. However, positive associations were observed between sFlt-1 and 1-PHE, and inverse associations were observed for PlGF with 2-NAP and 4-PHE. These shifts may be associated with the aberrant placentation believed to be characteristic of the development of preeclampsia. $^{28}$

Finally, regarding oxidative stress, PAH metabolites that were associated with 8-OHdG were also associated with increases in 8-iso, despite low correlation between the two biomarkers (Spearman correlation $<0.20$ ). ${ }^{22}$ Larger effect estimates were observed for 8-iso compared to 8-OHdG. The PAHs that showed the greatest effect estimates for association with these biomarkers were 2\&3-PHE, 1-PHE, and 1-PYR. Most PAH metabolites, however, were associated with increased 8-isoprostane except for 1-NAP and 4-PHE. Interestingly, these two compounds were associated with increases in the nitrosative stress biomarkers $\mathrm{ClY}$ and DiY, and the PAHs associated with increases in 8-OHdG and 8-iso were not. One possible explanation for these differences is that $8-\mathrm{OHdG}$ and 8 -iso reflect the impact of long-term exposure, while the nitrosative stress markers indicate acute effects. ${ }^{29}$

Data from in vitro studies, animal models, and epidemiologic studies using non-pregnant populations or other exposure/outcome assessment metrics support our findings of associations between exposure to PAHs and increases in circulating inflammation, adverse changes in angiogenic profiles, and increases in oxidative stress markers. Oxidative DNA damage is the best established mechanism of downstream PAH toxicity. PAHs are ligands for aryl hydrocarbon receptors, and binding to these receptors results in oxidative stress. ${ }^{30}$ Occupational studies demonstrate that exposure to PAHs, as assessed by urinary hydroxylated metabolites, is associated with increased 8-OHdG and 8-iso, and that women are potentially more susceptible to this damage. ${ }^{31,32}$ Studies of other populations at levels more similar to those observed presently have detected associations with these and other oxidative stress biomarkers as well. ${ }^{33,34}$ In regard to pregnant women specifically, this is the first study to examine associations between urinary PAH biomarkers and 8-OHdG, 8-iso, and the tyrosine adducts during gestation. Another study of 2-NAP and 1-PYR reported associations with increases in malondialdehyde, another biomarker of oxidative lipid peroxidation. ${ }^{35}$ Additionally, a study using biomarkers in urine collected at delivery observed significant positive association between 1-PYR and 8-OHdG. ${ }^{36} \mathrm{NY}$ in the placenta has been associated with particulate matter and other air pollutants, ${ }^{37}$ but to our knowledge this is the first study of urinary biomarkers of PAH exposure and these outcomes in humans.

Several studies have examined CRP and inflammatory cytokines in relation to PAH exposure, although not in pregnant women. A recent intensive study examining 280 cytokines as well as CRP in coke oven workers identified associations between most urinary PAH metabolite concentrations and CRP, although, as in our analysis, the association with 4- 
PHE was null. ${ }^{38}$ Similar to our study they did not observe associations between PAHs and IL-1 $\beta$, IL-6, IL-10, or TNF-a. ${ }^{38}$

Finally, no studies have examined the relationship between exposure to PAHs and the angiogenic biomarkers sFlt-1 and PIGF in humans. These biomarkers are predictive of preeclampsia, although whether they are a cause or an early marker remains unclear. ${ }^{39,40}$ Either way, the associations observed here between some urinary PAH metabolites and adverse angiogenic biomarker profiles suggest these exposures may be linked to decreased trophoblastic perfusion. The consequences of these effects (e.g., preeclampsia or intrauterine growth restriction) have been suggested in air monitoring studies but deserve further exploration in studies with urinary biomarkers of exposure. 9,41

This is one of few studies that examine urinary PAH metabolites in pregnant women, adding information on US women to the existing data from Canada, Poland, South Korea, and the Czech Republic. ${ }^{35,42-46}$ Urinary PAH metabolites reflect the internal dose from all recent exposure sources. Other studies of PAH exposure in pregnancy have examined PAH adducts in placenta, ${ }^{47}$ indicative of the biologically effective dose in that tissue. However, the focus is typically on benzo[a]pyrene adducts only. A limitation of our study was our use of spot urine samples to assess exposure. Little is known about how spot urine samples reflect exposure to PAH in pregnancy. Studies in rats and non-pregnant adults suggest that excretion occurs in 1-3 days following exposure. ${ }^{48}$ Thus, even if levels do not reflect exposure over all of gestation the time period of interest for the exposure-biomarker response is likely going to be captured. However, an expanded study is necessary to better understand how metabolite concentrations change over time and whether one sample reflects gestational exposure.

Another limitation of our study is the large number of comparisons made within a relatively small sample size. We chose to examine associations with all of the biomarkers we had available that may capture inflammation, perturbations in angiogenesis, and oxidative or nitrosative stress, as these pathways may be interrelated in pregnancy. We did not adjust for our multiple comparisons, nor refer to p-values in the interpretation of our results, as this was an exploratory study and meant to be hypothesis generating. Expanding these findings to a larger sample size is necessary to confirm these results, but, importantly, we highlight here some of the biomarkers that deserve further pursuit. Finally, as always in a crosssectional study, our study was limited in our ability to establish causality and some of the associations observed may have been due to reverse-causation.

In conclusion, we found evidence that urinary PAH metabolites are associated with increased inflammation and oxidative stress, as well as changes in angiogenic biomarker profiles, in pregnant women during the $3^{\text {rd }}$ trimester of gestation. The greatest effect estimates were observed for oxidative stress biomarkers, particularly 8-isoprostane, and for the inflammation marker CRP. No individual PAH metabolite or parent compound appeared to be uniformly associated with all of the biomarkers measured, suggesting that toxicities and mechanisms may differ by metabolite. Additional data is necessary to corroborate these findings and to clarify the impact of these changes on downstream pregnancy and/or developmental outcomes. 


\section{Supplementary Material}

Refer to Web version on PubMed Central for supplementary material.

\section{Acknowledgments}

We would like to thank Joel Whitfield of the University of Michigan Cancer Center Immunology Core for his analysis of CRP and the panel of inflammatory cytokines, and Elizabeth Hurst of Cayman Chemical for her analysis of 8-OHdG and 8-iso in urine. Funding for the original cohort study and analysis of sFlt-1 and PlGF biomarkers was provided by Abbott Diagnostics (9MZ-04-06N03). Funding for Dr. Kelly Ferguson was provided by the Intramural Research Program of the National Institute of Environmental Health Sciences, National Institutes of Health. Mass spectrometric measurement of tyrosine oxidation products were measured in Molecular Phenotyping Core, Michigan Nutrition and Obesity Center (P30DK89503). Other funding for this research project was provided by the National Institute of Environmental Health Sciences, National Institutes of Health (grants R01ES018872, P42ES017198, P01ES022844, P30ES017885, and P50ES026049).

\section{References}

1. Agency for Toxic Substances and Disease Registry. [accessed 3/17/17] Public health statement for polycyclic aromatic hydrocarbons (PAHs). Available from: https://www.atsdr.cdc.gov/ToxProfiles/ tp69-c1-b.pdf

2. Srogi K. Monitoring of environmental exposure to polycyclic aromatic hydrocarbons: a review. Environ Chem Lett. 2007; 5(4):169-195. [PubMed: 29033701]

3. Jung KH, Liu B, Lovinsky-Desir S, Yan B, Camann D, Sjodin A, Li Z, Perera F, Kinney P, Chillrud S. Time trends of polycyclic aromatic hydrocarbon exposure in New York city from 2001 to 2012: Assessed by repeat air and urine samples. Environ Res. 2014; 131:95-103. [PubMed: 24709094]

4. Perera F, Tang D, Whyatt R, Lederman SA, Jedrychowski W. DNA damage from polycyclic aromatic hydrocarbons measured by benzo [a] pyrene-DNA adducts in mothers and newborns from Northern Manhattan, the World Trade Center Area, Poland, and China. Cancer Epidemiology Biomarkers \& Prevention. 2005; 14(3):709-714.

5. Centers for Disease Control and Prevention. [accessed 3/17/17] Biomonitoring summary: Polycyclic aromatic hydrocarbons overview. Available from: https://www.cdc.gov/biomonitoring/ PAHs_FactSheet.html

6. Tonne CC, Whyatt RM, Camann DE, Perera FP, Kinney PL. Predictors of personal polycyclic aromatic hydrocarbon exposures among pregnant minority women in New York City. Environ Health Perspect. 2004; 112(6):754. [PubMed: 15121521]

7. Hecht SS. Tobacco carcinogens, their biomarkers and tobacco-induced cancer. Nature Reviews Cancer. 2003; 3(10):733-744. [PubMed: 14570033]

8. Kim KH, Jahan SA, Kabir E, Brown RJ. A review of airborne polycyclic aromatic hydrocarbons (PAHs) and their human health effects. Environ Int. 2013; 60:71-80. [PubMed: 24013021]

9. Choi H, Rauh V, Garfinkel R, Tu Y, Perera FP. Prenatal exposure to airborne polycyclic aromatic hydrocarbons and risk of intrauterine growth restriction. Environ Health Perspect. 2008; 116(5):658. [PubMed: 18470316]

10. Wilhelm M, Ghosh JK, Su J, Cockburn M, Jerrett M, Ritz B. Traffic-related air toxics and preterm birth: a population-based case-control study in Los Angeles County, California. Environ Health. 2011; 10(1):1. [PubMed: 21205326]

11. Perera FP, Rauh V, Whyatt RM, Tsai W-Y, Tang D, Diaz D, Hoepner L, Barr D, Tu Y-H, Camann D. Effect of prenatal exposure to airborne polycyclic aromatic hydrocarbons on neurodevelopment in the first 3 years of life among inner-city children. Environ Health Perspect. 2006:1287-1292. [PubMed: 16882541]

12. Edwards SC, Jedrychowski W, Butscher M, Camann D, Kieltyka A, Mroz E, Flak E, Li Z, Wang S, Rauh V. Prenatal exposure to airborne polycyclic aromatic hydrocarbons and children's intelligence at 5 years of age in a prospective cohort study in Poland. Environ Health Perspect. 2010; 118(9):1326. [PubMed: 20406721] 
13. Perera FP, Li Z, Whyatt R, Hoepner L, Wang S, Camann D, Rauh V. Prenatal airborne polycyclic aromatic hydrocarbon exposure and child IQ at age 5 years. Pediatrics. 2009; 124(2):e195-e202. [PubMed: 19620194]

14. Jedrychowski W, Galas A, Pac A, Flak E, Camman D, Rauh V, Perera F. Prenatal ambient air exposure to polycyclic aromatic hydrocarbons and the occurrence of respiratory symptoms over the first year of life. Eur J Epidemiol. 2005; 20(9):775-782. [PubMed: 16170661]

15. Rosa MJ, Jung KH, Perzanowski MS, Kelvin EA, Darling KW, Camann DE, Chillrud SN, Whyatt RM, Kinney PL, Perera FP. Prenatal exposure to polycyclic aromatic hydrocarbons, environmental tobacco smoke and asthma. Respir Med. 2011; 105(6):869-876. [PubMed: 21163637]

16. McElrath TF, Lim K-H, Pare E, Rich-Edwards J, Pucci D, Troisi R, Parry S. Longitudinal evaluation of predictive value for preeclampsia of circulating angiogenic factors through pregnancy. Am J Obstet Gynecol. 2012; 207(5):407. e1-407. e7. [PubMed: 22981320]

17. Ferguson KK, McElrath TF, Ko YA, Mukherjee B, Meeker JD. Variability in urinary phthalate metabolite levels across pregnancy and sensitive windows of exposure for the risk of preterm birth. Environ Int. 2014; 70:118-124. [PubMed: 24934852]

18. Onyemauwa F, Rappaport SM, Sobus JR, Gajdošová D, Wu Ra, Waidyanatha S. Using liquid chromatography-tandem mass spectrometry to quantify monohydroxylated metabolites of polycyclic aromatic hydrocarbons in urine. J Chromatogr B. 2009; 877(11):1117-1125.

19. Hornung RW, Reed LD. Estimation of average concentration in the presence of nondetectable values. Appl Occup Environ Hyg. 1990; 5(1):46-51.

20. Ferguson KK, McElrath TF, Meeker JD. Environmental phthalate exposure and preterm birth. JAMA pediatrics. 2014; 168(1):61-68. [PubMed: 24247736]

21. Ferguson KK, McElrath TF, Chen YH, Mukherjee B, Meeker JD. Longitudinal profiling of inflammatory cytokines and C-reactive protein during uncomplicated and preterm pregnancy. Am J Reprod Immunol. 2014; 72(3):326-336. [PubMed: 24807462]

22. Ferguson KK, McElrath TF, Chen Y-H, Loch-Caruso R, Mukherjee B, Meeker JD. Repeated measures of urinary oxidative stress biomarkers during pregnancy and preterm birth. Am J Obstet Gynecol. 2015; 212(2):208. e1-208. e8. [PubMed: 25111586]

23. Vivekanandan-Giri A, Byun J, Pennathur S. Quantitative analysis of amino Acid oxidation markers by tandem mass spectrometry. Methods Enzymol. 2011; 491:73. [PubMed: 21329795]

24. Centers for Disease Control and Prevention. [accessed 3/17/17] National Report on Human Exposure to Environmental Chemicals. Available from: https://www.cdc.gov/exposurereport/pdf/ fourthreport.pdf

25. Ferguson KK, McElrath T, Cantonwine D, Mukherjee B, Meeker J. Phthalate metabolites and bisphenol-A in association with circulating angiogenic biomarkers across pregnancy. Placenta. 2015; 36(6):699-703. [PubMed: 25913709]

26. White KL, Lysy HH, Holsapple MP. Immunosuppression by polycyclic aromatic hydrocarbons: a structure-activity relationship in B6C3F1 and DBA/2 mice. Immunopharmacology. 1985; 9(3): 155-164. [PubMed: 4040508]

27. Reynaud S, Deschaux P. The effects of polycyclic aromatic hydrocarbons on the immune system of fish: a review. Aquat Toxicol. 2006; 77(2):229-238. [PubMed: 16380172]

28. Wikström A-K, Larsson A, Eriksson UJ, Nash P, Nordén-Lindeberg S, Olovsson M. Placental growth factor and soluble FMS-like tyrosine kinase-1 in early-onset and late-onset preeclampsia. Obstet Gynecol. 2007; 109(6):1368-1374. [PubMed: 17540809]

29. Wannhoff A, Bölck B, Kübler A, Bloch W, Reuther T. Oxidative and nitrosative stress and apoptosis in oral mucosa cells after ex vivo exposure to lead and benzo [a] pyrene. Toxicol In Vitro. 2013; 27(2):915-921. [PubMed: 23318731]

30. Dalton TP, Puga A, Shertzer HG. Induction of cellular oxidative stress by aryl hydrocarbon receptor activation. Chem-Biol Interact. 2002; 141(1):77-95. [PubMed: 12213386]

31. Wang T, Feng W, Kuang D, Deng Q, Zhang W, Wang S, He M, Zhang X, Wu T, Guo H. The effects of heavy metals and their interactions with polycyclic aromatic hydrocarbons on the oxidative stress among coke-oven workers. Environ Res. 2015; 140:405-413. [PubMed: 25956561] 
32. Guo H, Huang K, Zhang X, Zhang W, Guan L, Kuang D, Deng Q, Deng H, Zhang X, He M. Women are more susceptible than men to oxidative stress and chromosome damage caused by polycyclic aromatic hydrocarbons exposure. Environ Mol Mutag. 2014; 55(6):472-481.

33. Farzan SF, Chen Y, Trachtman H, Trasande L. Urinary polycyclic aromatic hydrocarbons and measures of oxidative stress, inflammation and renal function in adolescents: NHANES 20032008. Environ Res. 2016; 144:149-157. [PubMed: 26610293]

34. Yang Q, Qiu X, Li R, Ma J, Li K, Li G. Polycyclic aromatic hydrocarbon (PAH) exposure and oxidative stress for a rural population from the North China Plain. Environmental Science and Pollution Research. 2015; 22(3):1760-1769. [PubMed: 25015715]

35. Kim H, Hwang J, Ha E, Park H, Ha M, Lee S, Hong Y, Chang N. Fruit and vegetable intake influences the association between exposure to polycyclic aromatic hydrocarbons and a marker of oxidative stress in pregnant women. Eur J Clin Nutr. 2011; 65(10):1118-1125. [PubMed: 21587280]

36. Al-Saleh I, Alsabbahen A, Shinwari N, Billedo G, Mashhour A, Al-Sarraj Y, Mohamed GED, Rabbah A. Polycyclic aromatic hydrocarbons (PAHs) as determinants of various anthropometric measures of birth outcome. Sci Total Environ. 2013; 444:565-578. [PubMed: 23314068]

37. Saenen ND, Vrijens K, Janssen BG, Madhloum N, Peusens M, Gyselaers W, Vanpoucke C, Lefebvre W, Roels HA, Nawrot TS. Placental Nitrosative Stress and Exposure to Ambient Air Pollution During Gestation: A Population Study. Am J Epidemiol. 2016; 184(6):442-449. [PubMed: 27601048]

38. Yang B, Deng Q, Zhang W, Feng Y, Dai X, Feng W, He X, Huang S, Zhang X, Li X. Exposure to Polycyclic Aromatic Hydrocarbons, Plasma Cytokines, and Heart Rate Variability. Sci Rep. 2016; 6:19272. [PubMed: 26758679]

39. Levine RJ, Maynard SE, Qian C, Lim K-H, England LJ, Yu KF, Schisterman EF, Thadhani R, Sachs BP, Epstein FH. Circulating angiogenic factors and the risk of preeclampsia. N Engl J Med. 2004; 350(7):672-683. [PubMed: 14764923]

40. Redman CW, Staff AC. Preeclampsia, biomarkers, syncytiotrophoblast stress, and placental capacity. Am J Obstet Gynecol. 2015; 213(4):S9. e1-4. [PubMed: 26428507]

41. Mendola P, Wallace M, Liu D, Robledo C, Männistö T, Grantz KL. Air pollution exposure and preeclampsia among US women with and without asthma. Environ Res. 2016; 148:248-255. [PubMed: 27085496]

42. Nethery E, Wheeler AJ, Fisher M, Sjödin A, Li Z, Romanoff LC, Foster W, Arbuckle TE. Urinary polycyclic aromatic hydrocarbons as a biomarker of exposure to PAHs in air: a pilot study among pregnant women. Journal of Exposure Science and Environmental Epidemiology. 2012; 22(1):70 81. [PubMed: 21915154]

43. Polanska K, Dettbarn G, Jurewicz J, Sobala W, Magnus P, Seidel A, Hanke W. Effect of Prenatal Polycyclic Aromatic Hydrocarbons Exposure on Birth Outcomes: The Polish Mother and Child Cohort Study. BioMed research international. 2014:408939. [PubMed: 25140312]

44. Polańska K, Hanke W, Sobala W, BrzeŸnicki S, Ligocka D. Predictors of environmental exposure to polycyclic aromatic hydrocarbons among pregnant women-prospective cohort study in Poland. Int J Occup Med Environ Health. 2011; 24(1):8-17. [PubMed: 21468898]

45. Wheeler AJ, Dobbin NA, Héroux M-E, Fisher M, Sun L, Khoury CF, Hauser R, Walker M, Ramsay T, Bienvenu J-F. Urinary and breast milk biomarkers to assess exposure to naphthalene in pregnant women: an investigation of personal and indoor air sources. Environ Health. 2014; 13(1): 1. [PubMed: 24405644]

46. Urbancova K, Lankova D, Rossner P, Rossnerova A, Svecova V, Tomaniova M, Veleminsky M, Sram RJ, Hajslova J, Pulkrabova J. Evaluation of 11 polycyclic aromatic hydrocarbon metabolites in urine of Czech mothers and newborns. Sci Total Environ. 2017; 577:212-219.

47. Manchester DK, Weston A, Choi J-S, Trivers GE, Fennessey PV, Quintana E, Farmer PB, Mann DL, Harris CC. Detection of benzo [a] pyrene diol epoxide-DNA adducts in human placenta. PNAS. 1988; 85(23):9243-9247. [PubMed: 3143115]

48. Jacob J, Seidel A. Biomonitoring of polycyclic aromatic hydrocarbons in human urine. J Chromatogr B. 2002; 778(1):31-47. 


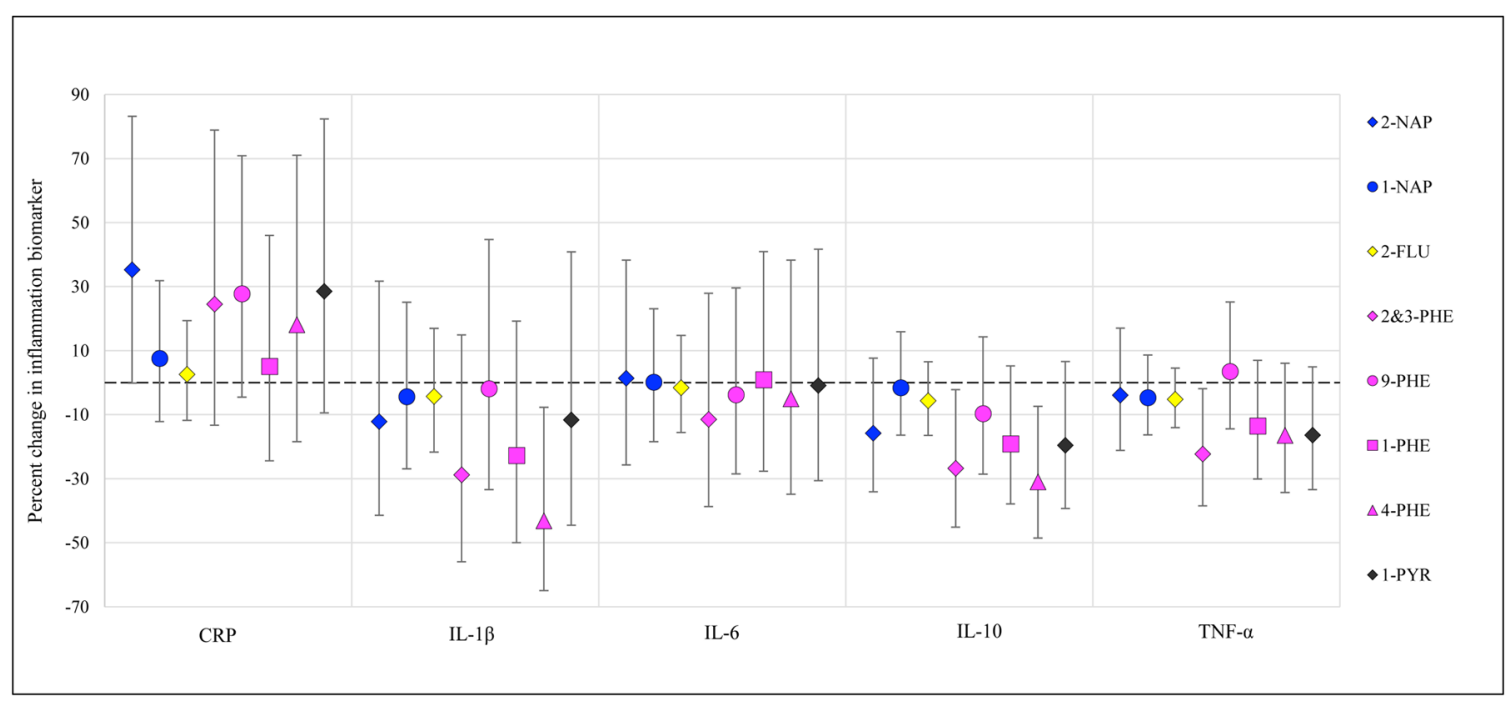

Figure 1.

Percent change in inflammation biomarker (95\% confidence intervals) in association with an interquartile range increase in uncorrected urinary PAH concentration. 


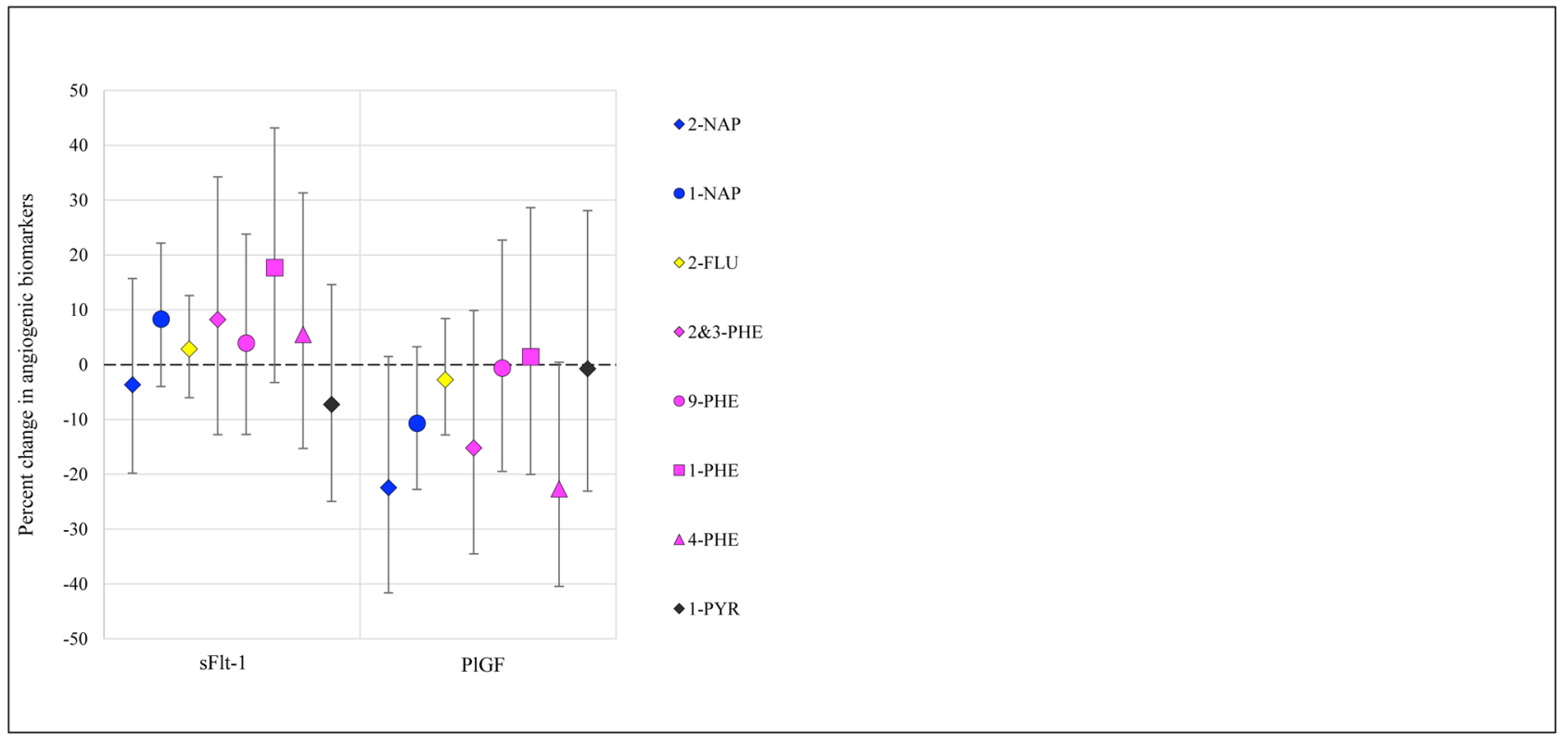

Figure 2.

Percent change in angiogenic biomarkers (95\% confidence intervals) in association with an interquartile range increase in uncorrected urinary PAH concentration. 


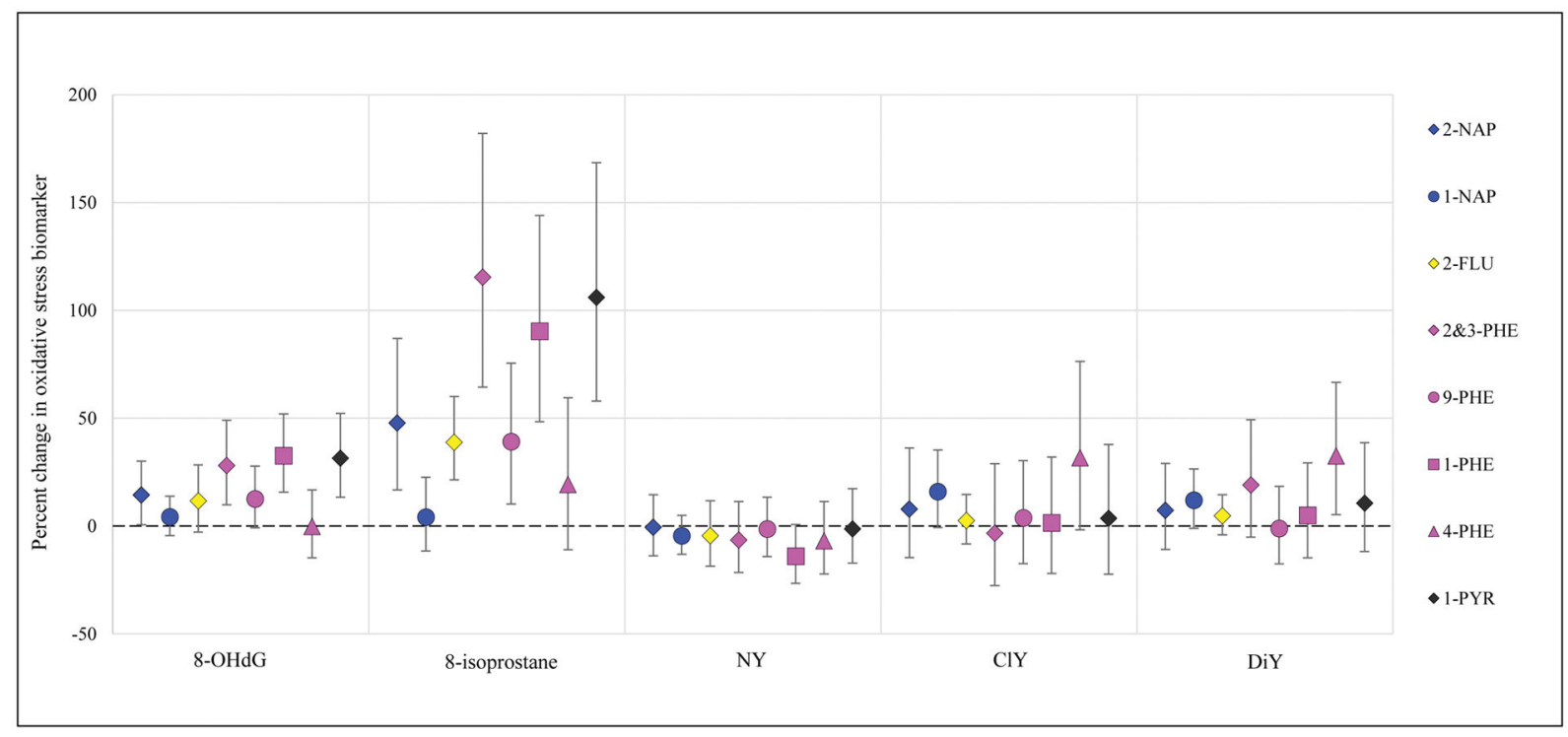

Figure 3.

Percent change in oxidative stress biomarkers (95\% confidence intervals) in association with an interquartile range increase in uncorrected urinary PAH concentration. 


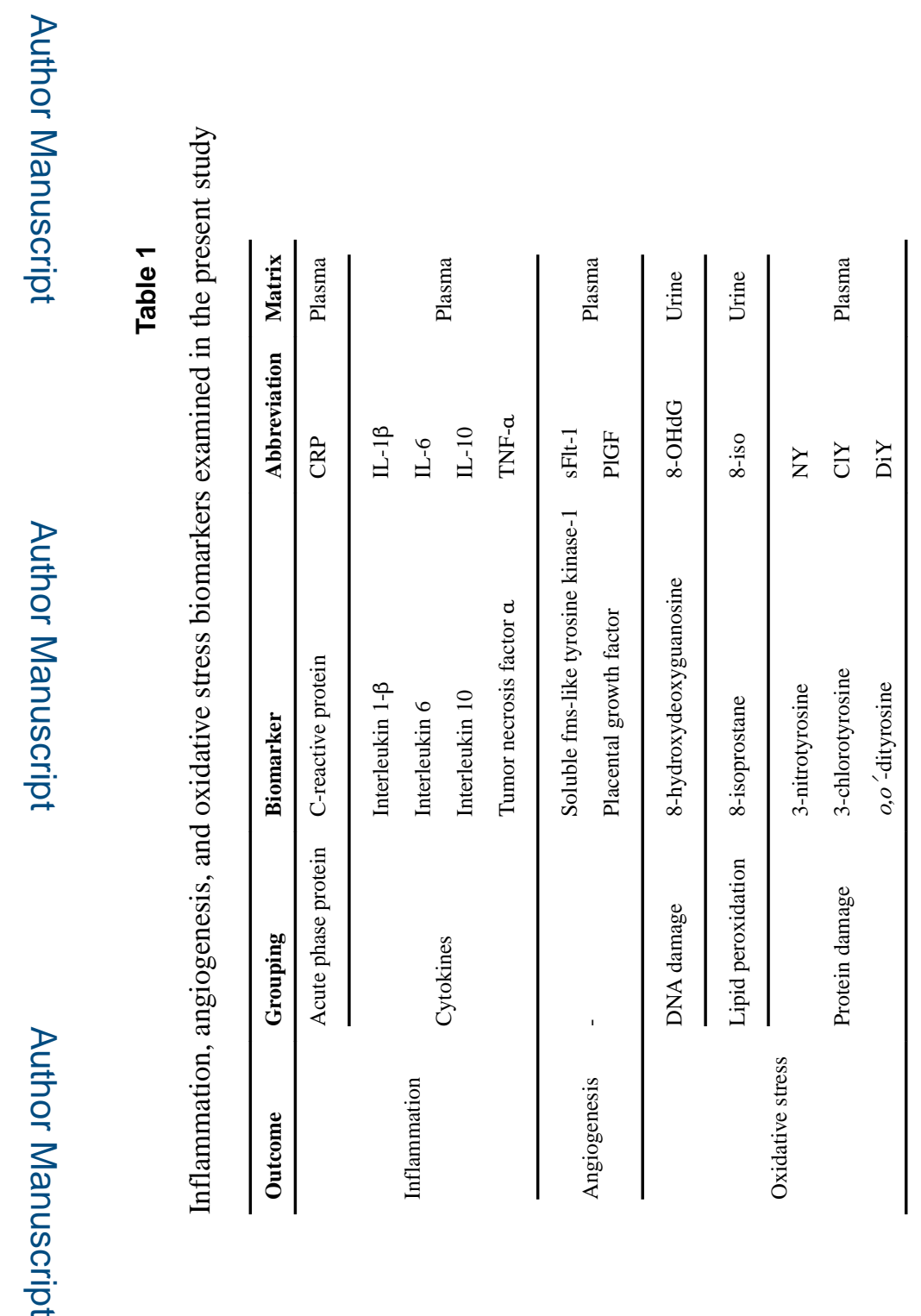




\section{Table 2}

Demographic characteristics of the study population

\begin{tabular}{|c|c|c|}
\hline Characteristic & & $\mathbf{N}(\%)$ \\
\hline \multirow{3}{*}{ Race/ethnicity } & White & $124(62 \%)$ \\
\hline & African-American & $25(12.5 \%)$ \\
\hline & Other & $51(25.5 \%)$ \\
\hline \multirow{4}{*}{ Education } & High school & $27(13.5 \%)$ \\
\hline & Technical school & $25(12.5 \%)$ \\
\hline & Junior/some college & $67(33.5 \%)$ \\
\hline & College graduate & $81(40.5 \%)$ \\
\hline \multirow{3}{*}{ Health insurance } & Private & $175(87.5 \%)$ \\
\hline & Public & $23(11.5 \%)$ \\
\hline & missing & $2(1 \%)$ \\
\hline \multirow{3}{*}{ BMI at visit 1} & $<25 \mathrm{~kg} / \mathrm{m}^{2}$ & $97(48.5 \%)$ \\
\hline & 25 to $<30 \mathrm{~kg} / \mathrm{m}^{2}$ & $56(28 \%)$ \\
\hline & $>30 \mathrm{~kg} / \mathrm{m}^{2}$ & $47(23.5 \%)$ \\
\hline \multirow{2}{*}{ Tobacco use in pregnancy } & No smoking & $184(92 \%)$ \\
\hline & Some smoking & $16(8 \%)$ \\
\hline \multirow{3}{*}{ Alcohol use in pregnancy } & No alcohol use & $189(94.5 \%)$ \\
\hline & Some alcohol use & $8(4 \%)$ \\
\hline & missing & $3(1.5 \%)$ \\
\hline \multirow{2}{*}{ Parity } & Nulliparous & $87(43.5 \%)$ \\
\hline & Parous & $113(56.5 \%)$ \\
\hline \multirow{2}{*}{ Fetal sex } & Male & $91(45.5 \%)$ \\
\hline & Female & $109(54.5 \%)$ \\
\hline \multirow{2}{*}{ Preterm } & No & $124(62 \%)$ \\
\hline & Yes & $76(38 \%)$ \\
\hline
\end{tabular}

Environ Sci Technol. Author manuscript; available in PMC 2018 April 18. 


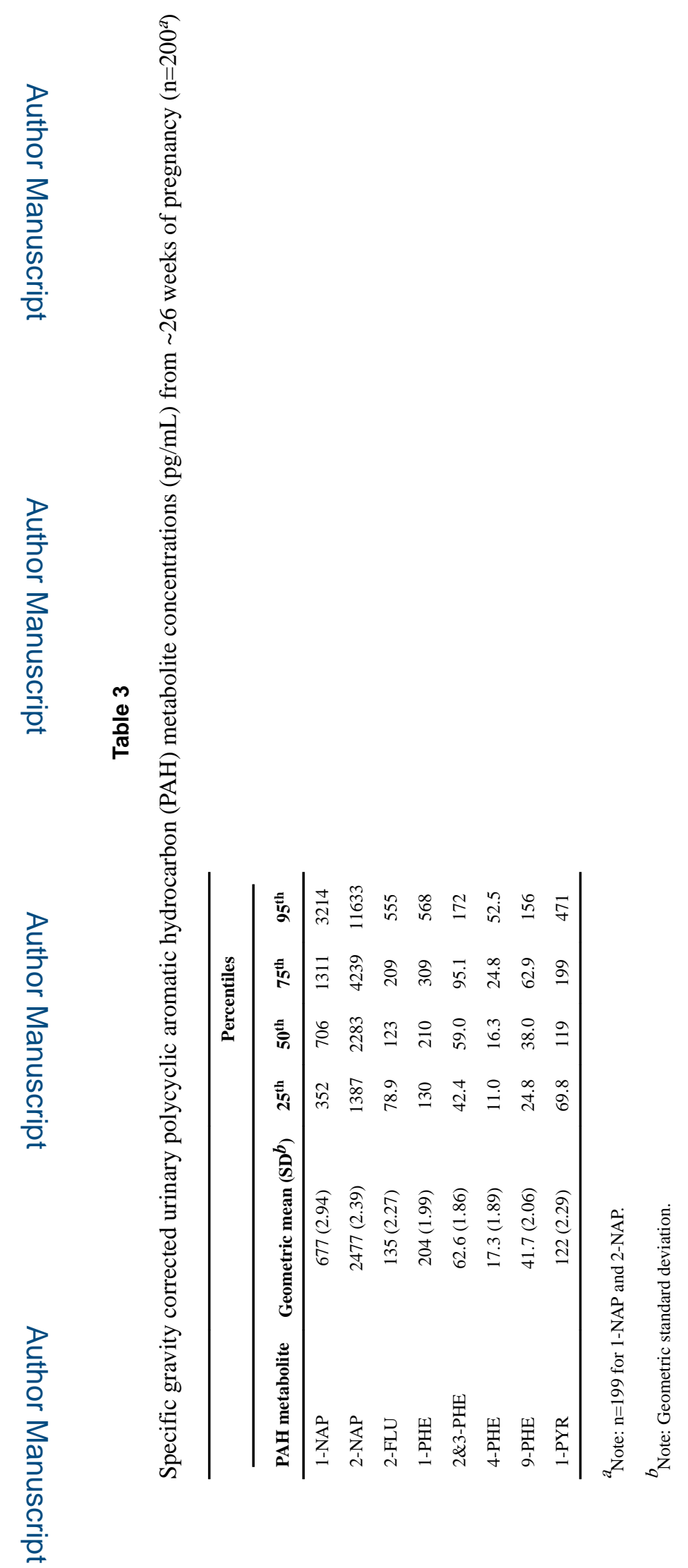

Environ Sci Technol. Author manuscript; available in PMC 2018 April 18. 


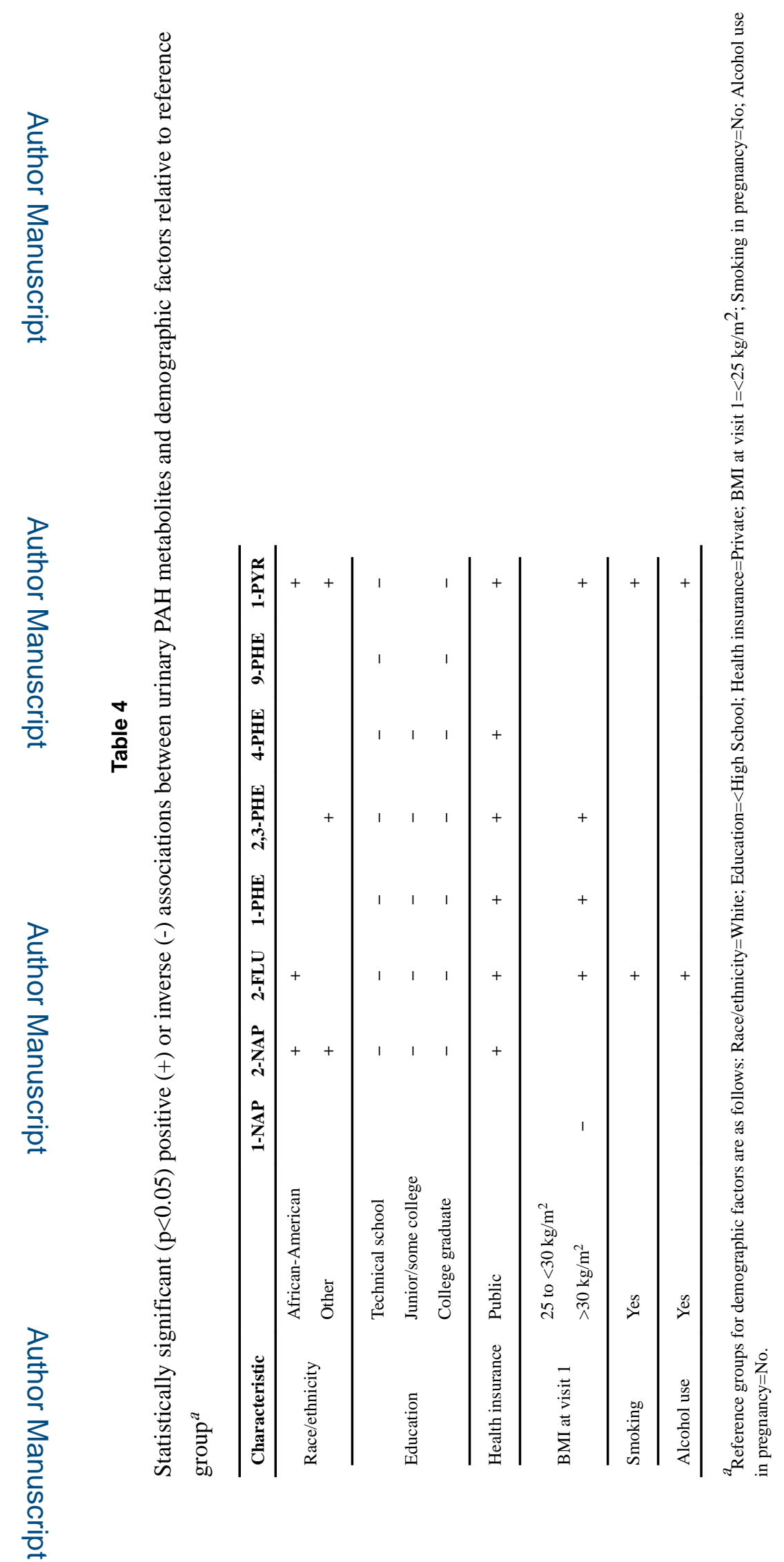

Environ Sci Technol. Author manuscript; available in PMC 2018 April 18. 


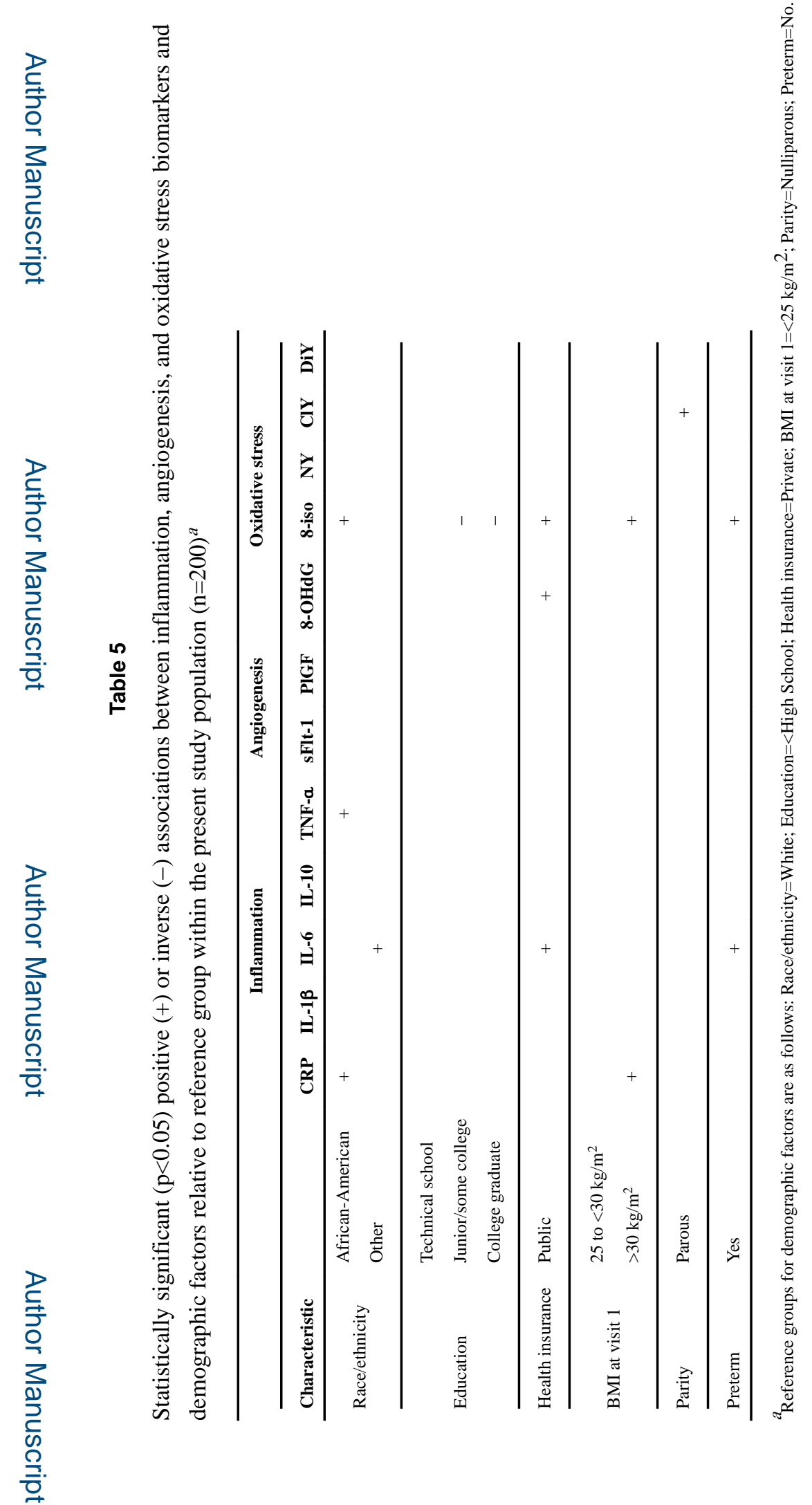

Environ Sci Technol. Author manuscript; available in PMC 2018 April 18. 